\title{
Variation de la réponse physiologique et agronomique du niébé (Vigna unguiculata L.Walp) à différentes dates de semis au Burkina Faso.
}

\author{
Adama Pascal KIHIND01* ${ }^{*}$, Hugues Roméo BAZIE¹, Razacswendé Fanta OUEDRAOG01, Pépin Patrice \\ SOME1, Edmond DONDASSE1, Gérard ZOMBRE¹, Koffi TOZO² \\ 'Université Ouaga I Pr Joseph Ki-Zerbo, Unité de Formation et Recherche en Sciences de la Vie et de la Terre, \\ Laboratoire Biosciences - 03 BP 7021 Ouagadougou 03, Burkina Faso \\ 2Université de Lomé Togo, Département de Botanique, 01 BP 1515 Lomé 01, Togo. \\ * Auteur correspondant ; E-mail : adama.passi@yahoo.fr et kihindoadamapascal@gmail.com , Téléphone : (+226) \\ $76044500 /(+226) 78300908$.
}

Original submitted in on 23 $3^{\text {rd }}$ August 2016. Published online at www.m.elewa.orgon $30^{\text {th }}$ November 2016 http://dx.doi.org/10.4314/jab.v107i1.1

\section{RÉSUMÉ}

Objectif : L'étude a eu pour objectif d'étudier l'effet des dates de semis au Burkina Faso sur les réponses physiologiques et agronomiques à travers l'évaluation de la transpiration, du potentiel hydrique foliaire et du rendement gousses et grains du Niébé (KVX 61.1).

Méthodologie et résultats : L'effet de différentes dates de semis du niébé sur sa physiologie a été étudié selon un dispositif en blocs complets randomisés à quatre répétitions. Les Différentes dates de semis ont eu un effet significatif $(p<0,0001)$ sur le taux de transpiration et le potentiel hydrique foliaire des plants. Le potentiel hydrique foliaire a été moins élevé et le taux de transpiration plus élevé au 40ème JAS aux dates de semis du 10 février, du 25 février et du 11 mars. Le rendement par plant a été affecté par une faible productivité des plantes issues de ces différentes dates de semis.

Conclusion et application : Les deux dates de semis, le 26 mars et le 10 avril sont des périodes qui permettent un développement optimal de la plante en culture de contre-saison chaude avec d'assez bon rendements. La variation des réponses physiologiques par rapport à la date de semis pourrait être utilisée comme critère d'adaptation (tolérance) à ces différentes dates de semis. La détermination des paramètres physiologiques pourrait être un indicateur pertinent de la période favorable de semis permettant d'orienter les agriculteurs qui désirent pratiquer la culture de la variété KVX 61.1 du niébé en culture de saison sèche par irrigation.

Mots-clés : Burkina Faso, niébé, potentiel hydrique, réponse physiologique, Transpiration

\section{INTRODUCTION}

La sécheresse sévit au sahel depuis les années soixante dix. Due principalement à la baisse de la pluviométrie et à une augmentation de sa variabilité spatio-temporelle, elle est devenue le principal facteur limitant de l'agriculture dans cette région
(Dancette, 1983). Responsable de la baisse et de la fluctuation des rendements des cultures, la sécheresse affecte les besoins alimentaires des populations sahéliennes à tel point que les besoins minimaux sont rarement satisfaits (Van et al., 1995). 

(Vigna unguiculata L.Walp) à différentes dates de semis au Burkina Faso.

Le Burkina Faso est l'un des pays où l'agriculture constitue l'activité principale avec plus de $80 \%$ de la population active. En dépit de cela, la production agricole actuelle n'arrive pas à couvrir les besoins alimentaires tant en quantité qu'en qualité. Ce paradoxe s'explique par la vulnérabilité des cultures aux aléas climatiques qui justifie la faiblesse des rendements. Cette vulnérabilité aux changements climatiques est aggravée à différents niveaux à savoir la dépendance de l'agriculture à la pluviométrie, la récurrence de la sécheresse, la pauvreté, la faible fertilité des sols, l'accroissement de la population et la faible capacité de résilience des populations ( Ozer et al., 2010). Le niébé (Vigna unguiculata (L.) Walp.) est une importante denrée de base en Afrique subsaharienne, particulièrement dans les savanes arides de l'Afrique de l'Ouest (Singh et al., 1997). Ses graines représentent une précieuse source de protéines végétales, de vitamines et de revenus pour I'homme. La culture du niébé au Burkina Faso confrontée à des contraintes climatiques comme le stress hydrique occasionné par une absence prolongée de pluie en saison pluvieuse. Malgré sa réputation de plante résistante à la sécheresse, les déficits hydriques récurrents dans les pays sahéliens dont le Burkina Faso, réduisent significativement la croissance et la

\section{MATERIELS ET METHODES}

Site expérimental : L'étude a été conduite dans le jardin expérimental de I'UFR/SVT situé à I'Université Ouaga 1 Pr Joseph Ki-Zerbo, au Burkina Faso en conditions naturelles d'éclairement, de température et d'hygrométrie. La parcelle expérimentale est située à une altitude de 319 mètres, $12^{\circ} 22^{\prime} 45,6^{\prime \prime}$ de latitude Nord et $01^{\circ} 29^{\prime} 52,3^{\prime \prime}$ de productivité du niébé (Muchero et al., 2008). Cependant une maitrise des potentialités de production de cette culture en saison sèche en fonction des dates de semis, pourrait être une stratégie de résilience des populations au système de cette spéculation aux nouvelles réalités climatiques. En effet, Useni et al. (2014), comparant trois périodes de semis du niébé en RD Congo (15 décembre, 30 décembre et 15 janvier), ont montré que la matière sèche et la production étaient élevées sur les parcelles ensemencées au 30 décembre. La variation d'un certain nombre de paramètres physiologiques dont : le taux de transpiration foliaire et le potentiel hydrique foliaire sont des indicateurs pertinents de la réponse des plantes à la sécheresse car ils sont l'une des premières fonctions de la plante affectée par le déficit hydrique (Choné et al., 2000) avec comme corrolaire une réduction des rendements (Van Damme, 1990). La présente étude vise (i) à évaluer les effets de différentes dates de semis en saison sèche sur la transpiration, le potentiel hydrique foliaire, (ii) à mieux comprendre l'influence de la variation de ces paramètres physiologiques sur la productivité du niébé au Burkina Faso et (iii) à déterminer la période de semis en saison sèche qui soit optimum pour une bonne productivité du niébé.

longitude Ouest. La pluviométrie annuelle de la zone est comprise entre 600 et $900 \mathrm{~mm}$ (Thiombiano et Kampmann, 2010). Le tableau 1 présente les paramètres climatiques du site d'étude durant la période expérimentale.

Tableau 1 : Moyennes de température et d'humidité relative

\begin{tabular}{|c|c|c|c|c|c|c|}
\hline Heures & \multicolumn{2}{|c|}{6 heures } & \multicolumn{2}{|c|}{13 heures } & \multicolumn{2}{|c|}{18 heures } \\
\hline Essais & $\mathrm{T}^{\circ} \mathrm{C}$ & HR\% & $\mathrm{T}^{\circ} \mathrm{C}$ & HR\% & $\mathrm{T}^{\circ} \mathrm{C}$ & HR\% \\
\hline 1er Essai & $24,48 \pm 3,87$ & $59,56 \pm 19,00$ & $40,28 \pm 3,96$ & $32,56 \pm 13,97$ & $32,59 \pm 3,18$ & $39,00 \pm 16,26$ \\
\hline 2ème Essai & $25,39 \pm 3,31$ & $64,91 \pm 19,95$ & $40,54 \pm 4,00$ & $36,66 \pm 16,06$ & $32,92 \pm 3,08$ & $43,23 \pm 18,48$ \\
\hline 3ème Essai & $25,92 \pm 3,22$ & $70,70 \pm 17,64$ & $40,35 \pm 3,96$ & $40,95 \pm 15,90$ & $33,02 \pm 3,02$ & $48,61 \pm 19,29$ \\
\hline 4ème Essai & $26,70 \pm 2,13$ & $77,84 \pm 12,22$ & $39,59 \pm 4,30$ & $46,89 \pm 14,12$ & $32,62 \pm 3,50$ & $56,17 \pm 17,96$ \\
\hline $5^{\text {sème Essai }}$ & $26,55 \pm 1,84$ & $82,59 \pm 9,39$ & $38,14 \pm 4,75$ & $52,76 \pm 15,07$ & $31,57 \pm 3,86$ & $63,02 \pm 18,04$ \\
\hline
\end{tabular}

$T^{\circ} \mathrm{C}=$ température en degré Celsius; $H R \%=$ humidité relative en pourcentage

Matériel végétal : L'étude a porté sur la variété KVX 61.1 Agronomique (INERA, station Kamboissin) au Burkina de niébé (Vigna unguiculata) à cycle court fournie par l'Institut de l'Environnement et de la Recherche Faso. La plante de niébé a un port érigé.. Elle fleurit à partir du 43 ème et du 45 ème jour après semis. Sa 
croissance est déterminée. Le sol utilisé est une terre enrichie en matière organique (fumier) Cette terre a préalablement été séchée à la température ambiante et tamisée à $2 \mathrm{~mm}$ (Tableau 2). La terre a ensuite été répartie dans des pots en plastique (diamètre supérieur
$22 \mathrm{~cm}$, diamètre inférieur $17 \mathrm{~cm}$ et profondeur $22 \mathrm{~cm}$ ). Chaque pot utilisé contenait six (06) $\mathrm{kg}$ de terre sèche. Le fond de chaque pot est a minutieusement été perforé et recouvert de papier mouchoir pour laisser égoutter l'eau après arrosage.

Tableau 2 : Caractéristiques chimiques du sol de culture fournies par le BUNASOL

\begin{tabular}{|l|l|l|l|l|l|}
\hline $\begin{array}{l}\text { Matière } \\
\text { organique totale }\end{array}$ & Carbone total & Azote total & Phosphore total & $\begin{array}{c}\text { Phosphore } \\
\text { assimilable }\end{array}$ & Potassium total \\
\hline $2,958 \%$ & $1,716 \%$ & $0,135 \%$ & $472,1 \mathrm{ppm}$ & $45,64 \mathrm{ppm}$ & $1701,02 \mathrm{ppm}$ \\
\hline
\end{tabular}

Source : Bureau National des Sols

Dispositif expérimental : Les essais ont été réalisés selon un dispositif en blocs complets randomisés à quatre répétitions à un facteur qui est la date de semis. Chaque bloc se compose de 18 pots dont 03 pots par unité expérimentale. Les semis ont été effectués à raison de 04 graines par pot. Un démariage à 1 plant par pot a été réalisé le 14ème jour après semis (JAS). La quantité d'eau apportée aux plantes à partir du 15 ème JAS a été de 1000 $\mathrm{ml}$ (Capacité au Champ du sol utilisé) par pot tous les deux jours (chaque 48 heures) car selon Kihindo et al, en 2015, l'arrosage tous les deux jours permet d'avoir un rendement optimal de KVX 61.1 en culture de saison sèche. Les pots ont été régulièrement désherbés. Ainsi, cinq (05) essais dont les protocoles expérimentaux sont identiques, espacés de quinze (15) jours en période chaude de février à avril 2012, ont été menés à savoir :

- $\quad$ Essai 1 : du 10 février 2012 au 20 mai 2012 ;

- $\quad$ Essai 2 : du 25 février 2012 au 04 juin 2012;

- $\quad$ Essai 3 : du 11 mars au 19 juin 2012 ;

- $\quad$ Essai 4 : du 26 mars au 04 juillet 2012 ;

- $\quad$ Essai 5 : du 10 avril au 19 juillet 2012.

Paramètres mesurés : Au cours de chaque essai, la température et l'humidité relative de l'air ont été enregistrées à l'aide d'un thermohygromètre de marque HANNA HI 9564 pour évaluer l'influence des facteurs environnementaux. Les valeurs ont été relevées à $6 h, 13$ $\mathrm{h}$ et $18 \mathrm{~h}$ quotidiennement (tableau 1). Pour évaluer l'influence des conditions climatiques qui ont prévalu sur le fonctionnement des plantes à ses différentes dates de semis, les paramètres physiologiques tels que le potentiel hydrique foliaire et la transpiration des plantes ont été mesurés. Le potentiel hydrique foliaire des plantes au stade végétatif et au stade floraison, avant leur alimentation hydrique, a été déterminé à partir du 40 ème JAS et à partir du stade $50 \%$ de floraison des plantes, en mesurant le potentiel hydrique foliaire de base $(\Psi b)$ et le potentiel hydrique foliaire minimal $(\Psi \mathrm{m})$ l'aide de la chambre à pression de Scholander (Model : DIK-7002, Measuring instrument, DaikirikaKogyoCo.LTD). Le potentiel de base $(\Psi b)$ a été mesuré avant l'aube (entre $3 \mathrm{~h} 30 \mathrm{~min}$ et $6 \mathrm{~h}$ ), au moment où la transpiration est presque nulle (Gebrekirstos et al., 2006). Le potentiel minimal $(\Psi \mathrm{m})$ a été mesurée en mi-journée (13h à15h). La transpiration de chaque plant a été précisément mesuré toutes les heures entre $6 \mathrm{~h}$ et $18 \mathrm{~h}$ à partir des pertes de poids de l'ensemble sol plus plante. La transpiration après arrosage a été déterminée, par la différence entre l'évapotranspiration (perte de poids des pots avec plantes) et l'évaporation (perte de poids des pots témoins sans plantes dont la surface est recouverte de paille pour reconstituer l'ombre des feuilles des plantes). Les pertes de poids des pots sont déterminées par la différence entre le poids d'un pot au temps $T_{1}$ et son poids au temps $T_{2}$. La durée entre $T_{1}$ et $T_{2}$ est d'une heure. Les pesées des pots ont été effectuées à l'aide d'une balance électronique de marque Sartorius de précision $0,001 \mathrm{~g}$. Pour évaluer le rendement des plantes aux différentes dates de semis, le nombre et le poids des gousses et des graines produites ont été régulièrement collectés.

Traitement des données et analyse statistique: L'ensemble des données a été (transpiration, potentiel hydrique, nombre et poids des gousses) soumis à une analyse de variance (ANOVA) avec comme facteur la date de semis à l'aide du logiciel XLSTAT version 7.5.2, logiciel utilisé également pour effectuer le test de signification des coefficients de corrélation de Pearson. La mise en évidence des différences significatives entre les moyennes a été réalisée au moyen de test du Student-Newman-Keuls (SNK) au seuil de $5 \%$ de probabilité.

\section{RÉSULTATS}


Paramètres physiologiques : Mesure de l'état hydrique des plantes (potentiel hydrique foliaire): Au stade végétatif, les potentiels hydriques de base des plantes sont significativement différents selon les dates de semis $(P=0,006)$ (Tableau3). Ainsi, Les valeurs des potentiels hydriques foliaires de base mesurées au 40ème JAS chez les plantes issues des semis du 25 février et du 11 mars, sont les plus basses avec respectivement $-0,975 \pm 0,13$ Mpa et $-0,763 \pm 0,024 \mathrm{Mpa}$ (Tableau3). Par contre, il n'y a pas eu de différence significative $(P=0,059)$ entre les valeurs de potentiel hydrique foliaire minimal des plantes des plantes aux différentes dates de semis (Tableau 3). Au stade floraison, l'analyse de variance a montré des différences significatives en fonction des dates pour le potentiel hydriques foliaire de base $(P=0,025)$ et minimal $(P=0,005)$ (Tableau 3). En outre, les potentiels hydriques foliaires de base et minimal des plantes issues des dates de semis du 10 février $\left(\Psi \mathrm{b}:-1,250 \pm 0,204 \mathrm{Mpa}\right.$ et $\Psi_{\mathrm{m}}$ :-

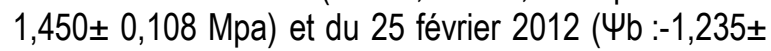
$0,191 \mathrm{Mpa}$ et $\left.\Psi_{\mathrm{m}}:-1,400 \pm 0,163 \mathrm{Mpa}\right)$ ont été significativement $(P=0,005)$ moins élevés au stade végétatif.

Transpiration : Les transpirations moyennes des plantes issues des semis du 10 février $\left(27 \pm 1,41 \mathrm{~g} \mathrm{~h}^{-1}\right)$, du 25 février $2012\left(25,667 \pm 2,14 \mathrm{~g} \mathrm{~h}^{-1}\right)$ sont significativement plus élevées que celles des plantes issues des semis du 11 mars $\left(19,833 \pm 1,10 \mathrm{~g} \mathrm{~h}^{-1}\right)$ et du 26 mars 2012 $\left(19,333 \pm 2,7 \mathrm{~g} \mathrm{~h}^{-1}\right)$ comme l'illustre la figure 1. La transpiration des plants a été significativement différente en fonction des heures de la journée (Figure 2). Au début de la journée, la transpiration est faible (entre $6 \mathrm{~h}$ et $9 \mathrm{~h}$ ), elle croit par la suite pour atteindre un maximum aux environs de $14 \mathrm{~h}$ puis décroit jusqu'à $18 \mathrm{~h}$.

\section{Paramètres agronomiques}

Nombre et poids des gousses et des graines: On note une différence significative du nombre de gousses $(P=0,002)$ en fonction des dates de semis. Par contre, aucune différence significative n'a été révélée pour le poids des gousses $(P=0,917)$ et le poids des grains $(P=0,133)$ (Tableau 4). Néanmoins, les valeurs moyennes du nombre de gousses, du poids sec des gousses et des graines par plant au 100ème JAS du niébé (KVX 61.1) ont augmenté du 10 février au 10 avril 2012 (Tableau 4). A $100 \mathrm{JAS}$, les plantes issues des semis du 10 février, du 25 février et du 11 mars ont eu un nombre de gousses moins important (respectivement, 13,50 \pm 3 gousses; $13 \pm 2,94$ gousses ; $11 \pm 1,41$ gousses) par rapport aux plantes issues des semis du 26 mars et du 10 avril avec respectivement $18,25 \pm 1,14$ gousses et $20,50 \pm 2,6$ gousses.

Corrélation entre les paramètres: La matrice de corrélation entre les paramètres mesurés (Tableau 5 ) montre que le potentiel hydrique foliaire de base au stade floraison est positivement corrélée $(r=0,990)$ avec la température à $13 \mathrm{~h}$ (heure la plus chaude) et la transpiration $(r=0,935)$. La transpiration est également positivement corrélé $(r=0,907)$ à la température (13h). Le nombre de gousse est négativement corrélé avec le potentiel de base au stade floraison $(r=-0,915)$, avec la transpiration $(r=-0,734)$ et avec la température à $13 \mathrm{~h}(r=$ $-0,909)$.

Tableau 3 : Potentiels hydriques foliaires de base et minimal au stade végétatif et au stade floraison (MPa).

\begin{tabular}{l|l|l|l|l}
\hline \multirow{2}{*}{ Date de semis } & Potentiel hydrique en Mpa au stade végétatif & \multicolumn{3}{|l}{ Potentiel hydrique en Mpa au stade floraison } \\
\cline { 2 - 5 } & $\Psi \mathrm{b}$ & $\Psi \mathrm{m}$ & $\Psi \mathrm{b}$ & $\Psi_{\mathrm{m}}$ \\
\hline 10-févr. & $-0,600 \pm 0,163^{\mathrm{a}}$ & $-1,075 \pm 0,061^{\mathrm{a}}$ & $-1,250 \pm 0,204^{\mathrm{b}}$ & $-1,450 \pm 0,108^{\mathrm{b}}$ \\
25-févr. & $-0,975 \pm 0,163^{\mathrm{b}}$ & $-1,325 \pm 0,265^{\mathrm{a}}$ & $-1,235 \pm 0,191^{\mathrm{b}}$ & $-1,400 \pm 0,163^{\mathrm{b}}$ \\
11-mars & $-0,763 \pm 0,024^{\mathrm{ab}}$ & $-1,200 \pm 0,040^{\mathrm{a}}$ & $-1,200 \pm 0,163^{\mathrm{b}}$ & $-1,160 \pm 0,130^{\mathrm{a}}$ \\
26-mars & $-0,663 \pm 0,162^{\mathrm{a}}$ & $-1,1000 \pm 0,122^{\mathrm{a}}$ & $-1,000 \pm 0,408^{\mathrm{ab}}$ & $-1,125 \pm 0,102^{\mathrm{a}}$ \\
10-avr & $-0,530 \pm 0,160^{\mathrm{a}}$ & $-1,020 \pm 0,097^{\mathrm{a}}$ & $-0,688 \pm 0,163^{\mathrm{a}}$ & $-1,100 \pm 0,163^{\mathrm{a}}$ \\
Probabilité & 0,006 & 0,059 & 0,025 & 0,005 \\
\hline
\end{tabular}

Les valeurs suivies par la même lettre dans la même colonne ne sont pas statistiquement différentes d'après le test de NewmanKeuls (SNK) au seuil de $5 \%$. b : potentiel hydrique foliaire de base, $\mathrm{m}$ : potentiel hydrique foliaire minimal.

Tableau 4 : Moyennes des paramètres de production des plantes en fonction du mois de semis

\begin{tabular}{l|c|c|c}
\hline Date de semis & Nombre de gousses & Poids des gousses(g) & Poids des graines(g) \\
\hline 10-févr. & $13,50 \pm 3.00^{\mathrm{bc}}$ & $10,91 \pm 3,39^{\mathrm{a}}$ & $9,78 \pm 1,63^{\mathrm{a}}$ \\
25-févr. & $13,00 \pm 2,94^{\mathrm{bc}}$ & $10,53 \pm 2,97^{\mathrm{a}}$ & $8,54 \pm 1,46^{\mathrm{a}}$ \\
11-mars & $11,00 \pm 1,41^{\mathrm{c}}$ & $9,63 \pm 3,12^{\mathrm{a}}$ & $7,57 \pm 1,30^{\mathrm{a}}$ \\
\hline
\end{tabular}


Kihindo et al., J. Appl. Biosci. 2016. Variation de la réponse physiologique et agronomique du niébé (Vigna unguiculata L.Walp) à différentes dates de semis au Burkina Faso.

\begin{tabular}{l|c|c|c}
\hline 26-mars & $18,25 \pm 1,40^{\mathrm{ab}}$ & $11,41 \pm 3,22^{\mathrm{a}}$ & $10,88 \pm 0,94^{\mathrm{a}}$ \\
10-avr & $20,50 \pm 2,60^{\mathrm{a}}$ & $11,89 \pm 4,92^{\mathrm{a}}$ & $11,84 \pm 1,02^{\mathrm{a}}$ \\
Probabilité & 0,002 & 0,917 & 0,133 \\
\hline
\end{tabular}

Dans une colonne, les valeurs ayant en commun une lettre ne sont pas significativement différentes au seuil (5\%) selon le test de Student Newman-Keuls.

Tableau 5 : Corrélation entre le potentiel hydrique foliaire de base, la transpiration, le nombre de gousses et la température à $13 \mathrm{~h}$.

\begin{tabular}{lllll}
\hline & $\Psi \mathrm{bFlo}$ & $\mathrm{Tr}$ & $\mathrm{Nb}$ de gousses & $\mathrm{T}^{\circ} \mathrm{C}(13 \mathrm{~h})$ \\
\hline$\Psi \mathrm{bFlo}$ & & & \\
$\mathrm{Tr}$ & 0,935 & & & \\
$\mathrm{Nb}$ de gousses & $-0,915$ & $-0,734$ & \\
$\mathrm{~T}^{\circ} \mathrm{C}(13 \mathrm{~h})$ & 0,990 & 0,907 & $-0,909$ & \\
\hline
\end{tabular}

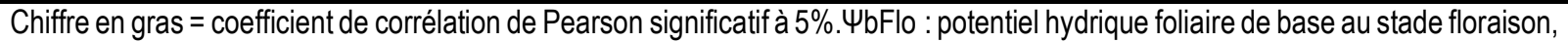
$\mathrm{Tr}$ : taux de transpiration, $\mathrm{Nb}$ de gousses : nombre de gousses, $\mathrm{T}^{\circ} \mathrm{C}(13 \mathrm{~h})$ : température à $13 \mathrm{~h}$

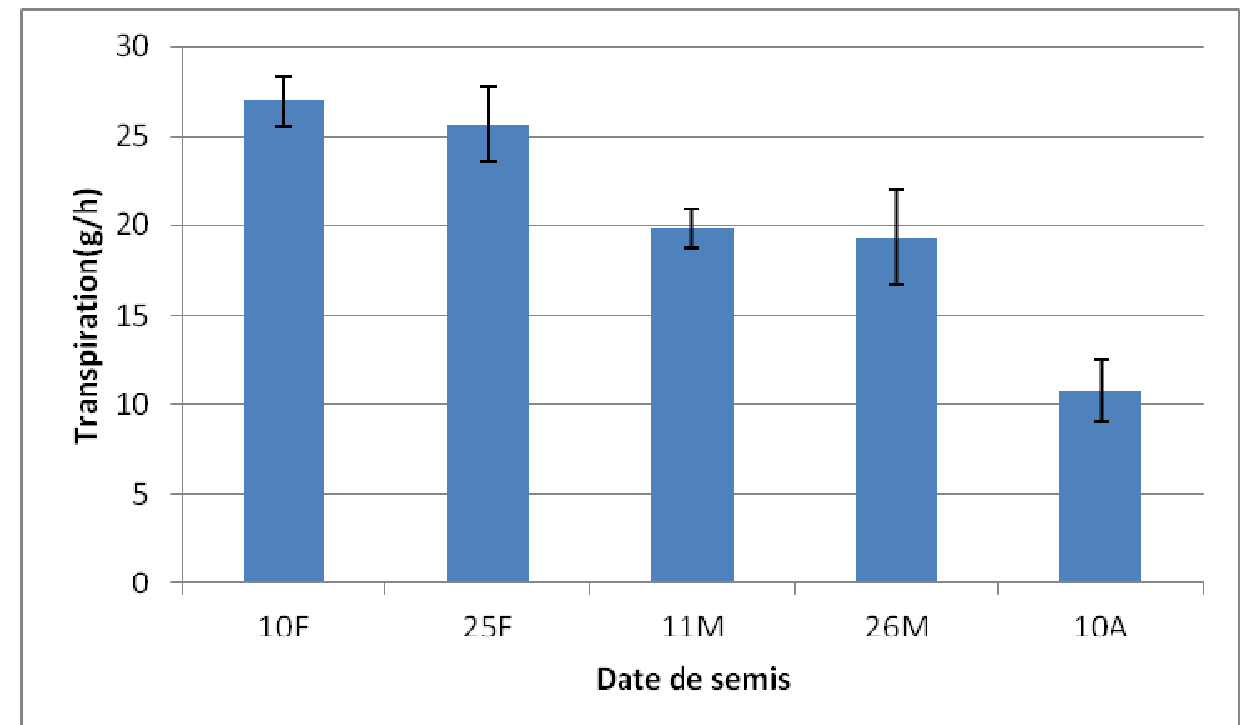

Figure 1 : Transpirations au 40ème JAS des plantes issues des semis du 10 février (10F), du 25 février (25F), du 11 mars (11M), du 26 mars (26M) et du 10 avril (10A). 


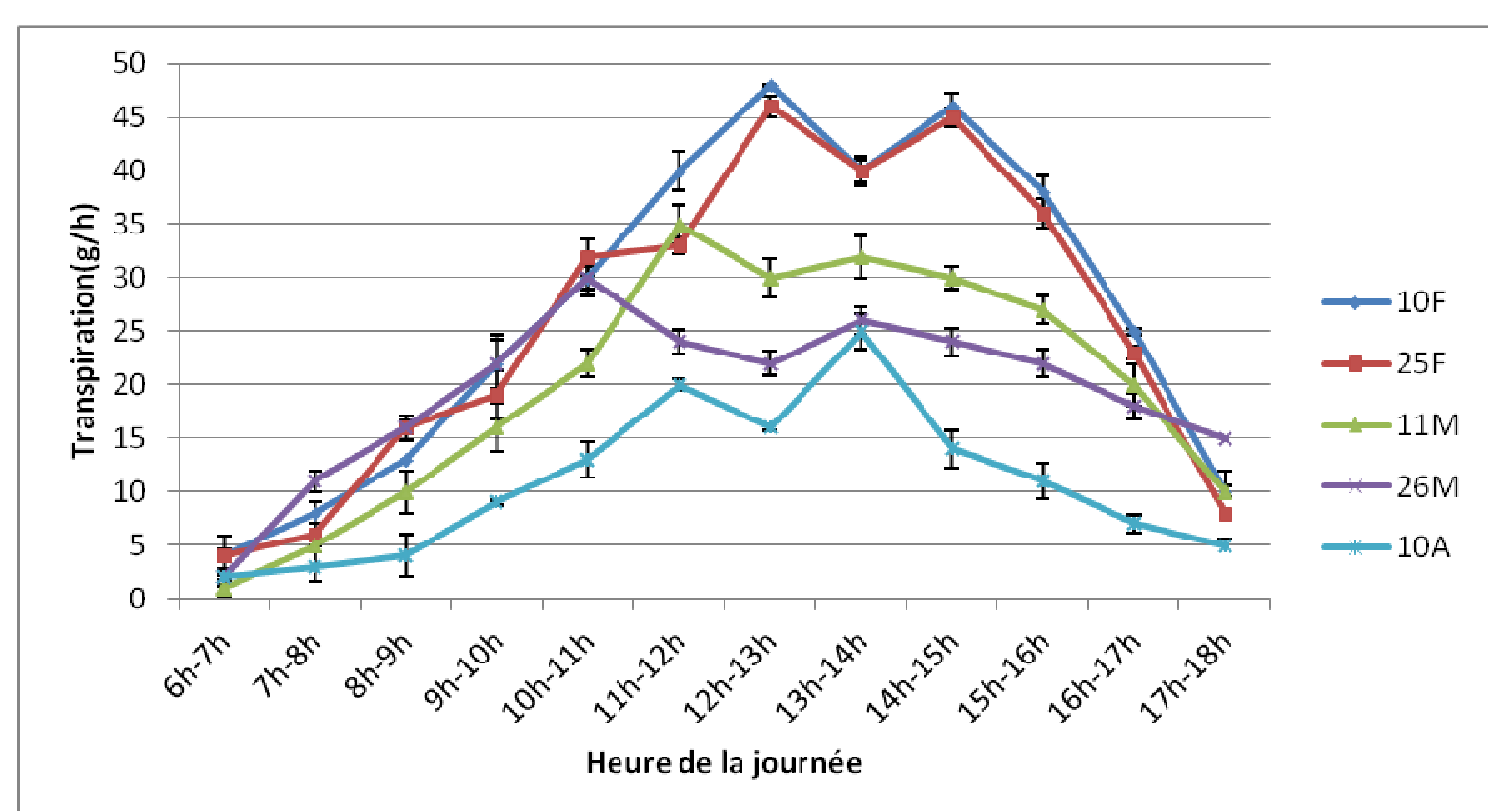

Figure 2 : Transpirations au 40ème JAS des plantes issues des semis du 10 février (10F), du 25 février (25F), du 11 mars (11M), du 26 mars (26M) et du 10 avril (10A) en fonction de l'heure de la journée.

\section{DISCUSSION}

Les moyennes de température et d'humidité mesurées pendant les 100 jours qu'a duré chaque essai, ont montré que les plantes issues des semis du 10 février, du 25 février et du 11 mars 2012 ont subi durant leur cycle semi-floraison, des températures élevées et une humidité relative faible. Cruz (2000) a observé que les plantes sont souvent sujettes à des stress environnementaux tels que les températures extrêmes, qui induisent un déficit hydrique. Zombré et al., en 2003 ont observé que les hautes températures provoquent un nombre important d'avortement des fleurs ce qui réduit le rendement des plantes. Le stress hydrique occasionné par la sècheresse de l'atmosphère qui a prévalu à ces dates de semis, a provoqué la mise en place d'un état de régulation hydrique de la plante qui se manifeste par la fermeture stomatique et par une régulation du potentiel osmotique (Brisson et Delecolle, 1992). Cette régulation du potentiel osmotique a conduit à une diminution du potentiel hydrique foliaire de base des plantes issues des semis du 10 février, du 25 février et du 11 mars au stade floraison. Cela s'est observé au stade floraison car les besoins en eau sont plus importants à ce stade pour assurer la formation des gousses de la plante. Somé et al., (2014) ont observé également que la variété de niébé KVX 61.1 (cultivée dans un sol fertilisé à l'aide de composts) stressée a un potentiel hydrique foliaire très faible. $\mathrm{Ce}$ faible niveau du potentiel hydrique foliaire de la plante suggérerait un ajustement osmotique des cellules dans le but de continuer à prélever le peu d'eau disponible dans les couches profondes du sol, afin de poursuivre son activité physiologique en dépit de la contrainte hydrique du sol. L'ajustement osmotique est généralement considéré comme un élément important dans la tolérance des plantes au stress hydrique (Bajji et al., 2001). Mais cet ajustement osmotique qui a conduit à la diminution du potentiel hydrique foliaire, a réduit la productivité (nombre de gousses) des plantes issues des dates de semis du 10 février, du 25et du 11 mars 2012. Brodribb et Holbrook (2003) ont constaté que la diminution du potentiel hydrique foliaire entraine la fermeture des stomates chez des plantes comme Simarou baglauca et Quercus oleoides, réduisant ainsi la photosynthèse et la productivité. Le test de corrélation révèle également que plus la température est élevée, plus le potentiel hydrique de base au stade floraison des plantes est moins élevé avec une forte transpiration. Les plantes issues des semis du 10 avril 2012 ont eu au stade floraison, les potentiels hydriques foliaires de base les plus élevés. Ces constats s'expliqueraient par le fait que les plantes issues des semis du 10 avril 2012, ont subi à partir du 40ème JAS une température moins élevée avec une forte humidité de l'air ambiant. Ce contexte a réduit la transpiration des plantes, d'où par conséquent, l'augmentation du potentiel hydrique foliaire de base. La transpiration des plantes mesurée à partir du 40ème JAS après arrosage, nous montre que les plantes issues des semis du 10 février, du 25 février 
2012 transpirent significativement $(P<0,0001)$ plus que les plantes issues des semis du 11 mars, 26 mars et du 10 avril 2012. La diminution de l'humidité relative, rendant l'air plus sec, abaisse considérablement le gradient de potentiel hydrique atmosphérique ce qui contribue également à augmenter la transpiration. Selon Wiley et Sons en 1997, les températures, plus fortes augmentent la transpiration en changeant le déficit de vapeur d'eau à la surface des feuilles. Une transpiration élevée est nécessaire au maintien de la température des feuilles sous des valeurs non létales (Cornic et al., 1999). Mais cette forte transpiration des plantes observée la journée, selon Durand (2007) dégrade le bilan entre la perte d'eau par transpiration et l'absorption d'eau du sol par les racines. De plus, Prat (2004) a constaté que si le temps devient chaud et sec, le potentiel hydrique de l'atmosphère (humidité relative de l'air très faible) diminue encore et les pertes dues à la transpiration ne sont plus compensées par l'absorption. Selon Compaoré, (2011) si l'évapotranspiration potentielle est supérieure à la quantité d'eau reçue par la plante, elle subit un stress hydrique entrainnant entre autre la baisse de rendement. Cette baisse de rendement s'est constatée ici par la corrélation négative entre le nombre de gousses produites et le taux de transpiration de la plante car plus la plante transpire, moins elle produit de gousses. Ceci expliquerait la faible productivité en gousses des plantes semées à ces périodes (10 février et 25 février 2012). Le 40 ème JAS des plantes issues des semis du 11 mars, du 26 mars et du 10 avril 2012 coïncide avec la période d'étude où les plantes ont moins transpiré. Nous pouvons conclure à travers ces constats que lorsque la température est moins élevée et l'humidité relative en hausse, le gradient de pression de vapeur entre la feuille et l'air environnant diminue, ce qui diminue la transpiration des plantes. La corrélation positive entre la température et le taux de transpiration de la plante (moins la température est élevée, moins la plante transpire) confirme ces observations. Bayles et al., (1994) ont observé également que le flux de transpiration est lié

\section{CONCLUSION}

La température et l'humidité relative de l'air sont des facteurs abiotiques faisant partie intégrante de l'environnement de la plante. L'étude a mis en évidence une forte influence de ces facteurs environnementaux sur les réponses physiologiques de la variété KVX 61.1, qui s'est traduite par une forte transpiration et un faible potentiel hydrique des plantes issues des semis du 10 et du 25 février 2012. De plus, l'étude a révélé également, l'effet des paramètres physiologiques sur la productivité au déficit d'humidité relative et que si le déficit de saturation est faible ('humidité de l'air élevée), la plante du rosier transpire peu. La réduction du taux de transpiration améliore l'état hydrique des tissus car les racines continuent à absorber l'eau alors que la transpiration est réduite (Wilkinson et Davies, 1997) ce qui a favorisé un fonctionnement optimal de la plante à ces périodes avec une production élevée de gousses. $P a r$ ailleurs, les plantes ont significativement $(P<0,0001)$ plus transpiré pendant les périodes assez chaudes de la journée et ont diminué leur transpiration (fermeture partielle des stomates correspondants à la «dépression de midi») durant les périodes les plus chaudes de la journée. Darrigan et al., en 1991, ont observé également «une dépression de midi» chez la Lierre terrestre lorsque les seuils de transpiration sont atteints lors d'une journée chaude et/ ou sèche ce qui permet à la plante d'éviter un stress hydrique. Les valeurs moyennes du nombre de gousses produit par plant, de leur poids sec et celui de leur graine jusqu' au 100ème JAS, ont augmenté progressivement pour les semis du 10 février au 10 avril 2012 avec une différence significative concernant les dates de semis. Cette augmentation progressive de productivité, correspond à l'abaissement progressif de la température, jusqu'à l'atteinte des températures optimales pour la réalisation des réactions métaboliques. La diminution progressive de la transpiration a pour conséquence la diminution progressive de la fermeture des stomates, d'où l'augmentation progressive des échanges gazeux (absorption de $\mathrm{CO}_{2}$ et rejet de $\mathrm{O}_{2}$ ), qui accroit par conséquent, progressivement la photosynthèse. L'accroissement progressif de la production des substances carbonées par la photosynthèse, va contribuer à l'augmentation progressive de la production de gousses. Une assez forte productivité par plant a été observée chez les plantes issues des semis du 26 mars et du 10 avril 2012 à cause de l'humidité relative atmosphérique élevée qui aurait permis une transpiration en adéquation avec l'absorption d'eau.

de la variété KVX61.1, qui s'est manifesté par le fait que, plus la plante transpire, plus elle possède un potentiel hydrique foliaire au stade floraison négatif et moins elle produit de gousses. Au regard de ces résultats, la période de culture de contre saison chaude permettant l'esquive qui consiste ici, à placer le cycle cultural pendant des périodes où la demande climatique est faible, c'est à dire avec une transpiration et un potentiel hydrique optimal 
après $40 \mathrm{JAS}$, et qui intervient à partir du 26 mars de

\section{REFERENCES BIBLIOGRAPHIQUES}

Bajji M, Kinet JM Etlutts S, 2001. The use of the electrolyte leakage method for assessing cell membrane stability as a water tolerance test in durum wheat. Plant Growth Regul.00 :1-10.

Bayles KA,Tomoeda CK, 1994. The functional linguistic communication inventory. Austin, TX: ProEd.In : The MIT encyclopedia of communication disorders. Edited by Raymond D.K. 625p.

Brisson N, et Delecoller R, 1992. Développement et modèles de simulation des cultures. Agronomie, 3: 253-263.

Brodribb TJ, Holbrook NM, 2003. Stomatal closure during leaf dehydration, correlation with other leaf physiological traits. Plant Physiol 132: 21662173.

Choné X, Tregoat O, Van LC, Dubourdieu D, 2000. Déficit hydrique modéré de la vigne : Parmi les 3 applications de la chambre à pression, la potentielle tige est l'indicateur le plus précis. J. int. Sci. Vigne vin, 34: 169-176.

Compaoré E, 2011. Effet du stress hydrique sur la croissance et la production du sésame (Sesamum indicum). Journal of Applied Biosciences. 37: 2460-2467.

Cruz de Carvalho HM, 2000. Étude physiologique, biochimique et moléculaire de la réponse à la sécheresse chez Phaseolus vulgari L. et Vigna unguiculata L. Walp. Implication de l'aspartique protéinase. Mise au point de l'étape préalable à la transgénèse: régénération in vitro des plantes. Thèse de Doctorat., Université Paris VI, $180 \mathrm{p}$.

Dancette N, 1983. Contraintes pédoclimatiques et adaptation de l'agriculture à la sécheresse en zones intertropicales, In: La sécheresse en zone intertropicale pour une lutte intégrée, CIRA-GERDAT-ISRA, pp. 27-39.

Darrigan JM, et Turck M, 1991. Étude expérimentale de la transpiration des végétaux. L'informatique scientifique dans l'enseignement de la biologie et de la géologie au lycée, INRP, pp181-183.

Durand JL, 2007. Les effets du déficit hydrique sur la plante: aspects physiologiques. Fourrage.190:181-195.

Gebrekirstos A, Teketay D, Fetene M, Mithohner R, 2006. Adaptation of five co-occurring tree and shrub species to water stress and its implication l'année.

in restoration of degraded lands for EcolManag $229: 259-267$

Kihindo AP, Bazié HR, Ouédraogo FR, Somé PP, Zombré G, Tozo K, 2015. Effet de la date de semis et du régime hydrique sur la réponse agromorphologique de deux varieties de niébé (KN1 et KVX 61.1) au Burkina Faso. International Journal of Innovation and Applied Studies. 12 :564-573.

Muchero W, Ehlers JD, Roberts PA, 2008. Seedling stage drought-induced phenotypes and droughtresponsive genes in diverse cowpea genotypes. Crop Science 48: 541-552.

Ozer P, Hountondji YC, Niang AJ, Karimoune S, Manzo OL, Salmon M, 2010. "Désertification au sahel : historique et perspectives". BSGLg.54 : 69-84.

Prat R, 2004. Adaptation des plantes aux climats secs. http://WWW.futura-sciences.

Com/magazines/environnement/infos/dossiers/d/ climatologie-adaptations-plantes-climats-secs476p.

Singh BB, Chanibliss OL, Sharma B, 1997. Récent advances in Cowpea breeding. In Advances in Cowpea Research- (Singh BB, Mohan Raj, Dashiell KE Jackai L E N. eds)-IITA. Ibadan, Nigeria, pp. 30-49.

Somé PP, Hien E, Tozo K, Zombré G, et Dianou D, 2014. Effets de six composts sur les réponses physiologiques, biochimiques et agronomiques du niébé Vigna unguiculata L. Walp var. KVX 61.1 au déficit hydrique. IJBCS. 1:31-45.

Thiombiano A, Kampmann D, 2010. Atlas de la biodiversité de l'Afrique de l'Ouest, Tome II: Burkina Faso, Ouagadougou et Frankfurt/Main.

Useni SY, Mayele K, Kasangij AKP, Nyembo KL, 2014.Effets de la date de semis et des écartements sur la croissance et le rendement du niébé (Vigna unguiculata L. Walp) à Lubumbashi, RD Congo". International Journal of Innovation and Applied Studies.6 : 40-47.

Van Damme PLJ, 1990. Adaptation to drought stress in plants. Med. Fac. Laudbouw. Rijks Univ. Gent. $55: 121-126$.

Van OEJ, Bidiger FR, Mahalakshmi V, Rao KP, 1995. Effect of water availability pattern on yield of pearl millet in semi-arid tropical environments. Euphytica, 89: 165-173. 
Wiley J, \& Sons ; FAO, 1997. Changement du climat et production agricole. Effets directs et indirects du changement des processus hydrologiques, pédologiques et physiologiques des végétaux, in: global climate change and agricultural production. Bazzaz F.A., Wim G., ISBNO-47195763-1.

Wilkinson S, Davies WJ, 1997. Xylem sap pH increase: a drought signal received at the apoplastic face of guard cell that involves the suppression of saturable abscisic acid uptake by epidermal symplast. Plant physiology, 113: 559-573.

Zombré G, Hema D, Falalou H, Sankara P, 2003. Influence des fortes températures diurnes sur la productivité du niébé en culture de contre saison. Sécheresse 4: 227-233. 